\title{
A Research Paper on Ameliorating the Lift-Off Efficiency of an Aerodyne
}

\author{
Kavish Soningra
}

\begin{abstract}
In this paper, I propose a peculiar model for the aerodyne wing. The principal aim of the model is to maximize the lift-off efficiency of aircrafts and to increase the effectiveness of in-flight maneuvers. The sudden, sharp movement of a leading-edge downward-movable control surface in the main wing (called "S-flap" in the paper) could significantly help increase the lift force, the upward acting force of an aircraft, working independently of an elevator located on the tail section of the craft. Finally, the advantages of the model are discussed.
\end{abstract}

Index Terms- S-FLAP, Lift-off Efficiency, Aerodynes, Flight, Aileron, Lift \& Drag Force.

\section{INTRODUCTION}

\section{A. Fundamentals of flight:}

Firstly, let us start by discussing the fundamentals of flight. An aircraft flies with the help of movable control surfaces and the flow of air around it. The wing is designed such that the air above the wing would flow faster than the air below, and thus create a region of low air pressure above the wings generating a lift force upward. Four forces act on an airplane. The weight of the plane acting downwards is balanced by the lift force generated by the craft's wings. The thrust of the engines that tends to move the aircraft forward is balanced by air resistance, or drag-produced by the resistance of the air to the forward motion of the airplane.To fly a craft all we need to do is produce more thrust than drag and more lift than its weight acting downwards. Thrust is simple to comprehend: The engines spin to create a region of low pressure just before them. This causes the air to rush in, which is then passed through a compressor and further ignited with the fuel in the combustion chamber. This same air is then passed through the turbine station, causing the first-stage fan to spin faster and take in much more air. This incoming air pushes the ignited air out from the exhaust. This backward push imparts the necessary forward thrust (the reaction force), which drives the aircraft at a speed of about 170 knots on the runway ground. $(1 \mathrm{knot}=0.514444 \mathrm{~m} / \mathrm{s})$

$B$. What about the lift force?

Every craft of course has wings to generate them. Any air traveller is aware of the huge surface area of the main wing. The wings are aerodynamically shaped to maximize the lift force. The airfoil shape of the wing curves the air flowing above it to move faster, and the air flowing below it slower, creating a difference in air pressure above and below - with a greater pressure below. This is responsible for producing the

Kavish Soningra, The Cathedral and John Connon School, Mumbai, India force that pushes the wings upward.

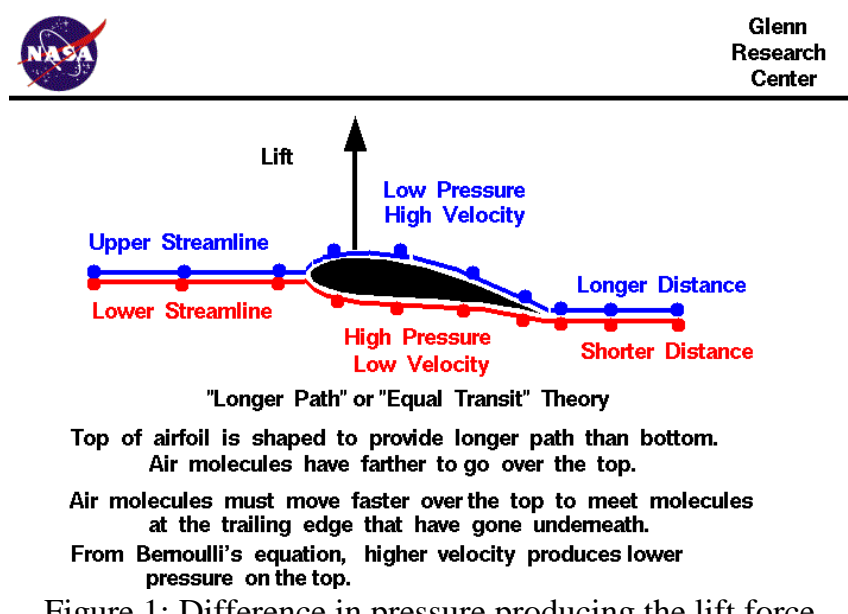

Figure 1: Difference in pressure producing the lift force.

Air is a fluid. When a fluid is in motion, it must move such that its mass is retained. Mass conservation places restrictions and limitations on the velocity field. [4]Let us consider the stationary flow of fluid through a duct (i.e., the inlet and outlet flows don't vary with time). The inflow and outflow are one-dimensional, so that the velocity $v$ and density $\rho$ are constant over the area $A$.

Applying the principle of mass conservation:

Since there is no flow through the side walls of the duct, the mass that comes in over the area of the inlet portion $A 1$ goes out of the area of the outlet portion $A 2$. The flow is constant so that mass is not cumulated. Over a short time-interval $\Delta t$,

Volume of inflow, $A 1=A 1 * v 1 * . \Delta t$

Here $v 1$ is the fluid velocity when it is flowing through $A 1$. Velocity * time gives distance (In this case, in the horizontal direction component); which, when multiplied with the two-dimensional area of the cross-section, gives the volume of the inflow.

Similarly,

Volume of outflow, $A 2=A 2 v 2 \Delta t$

Here $v 2$ is the fluid velocity when it is flowing through $A 2$.

We know that:Density=Mass/Volume.

Mass in $=\rho A 1 * v 1 * \Delta t$

Mass out $=\rho A 2 * v 2 * \Delta t$

By the law of conservation of mass, $\rho * A 1 * v 1 * \Delta t=\rho * A 2 * v 2 * \Delta t \rightarrow A 1 * v 1=A 2 * v 2$

This equation stated above is for steady one-dimensional fluid flow. It shows that the larger the area, the lower the velocity and inverse. This is exactly why water from a pipe rushes out at a greater velocity if the mouth of the pipe is 
pinched (surface area decreased). This very principle is used by aircrafts too.

Nonetheless, it should be noted that the equal transit theory is flawed since the air that flows above and below the wing need not meet at the trailing edge-"the trailing edge of an aerodynamic surface such as a wing is its rear edge, where the airflow separated by the leading edge meets." Additionally, in contrast to what is stated in the equal transit theory, the difference in velocity need not give rise to the pressure difference, rather the opposite. Actually, as the streamline curvature theory suggests, the curvature of the wing is indeed responsible for generating the required lift force. The key is to understand that if a streamline is curved, there must be a pressure gradient across the streamline, with pressure increasing in the direction away from the center of curvature.

The fluid flow is steady at any given point, if the velocity of each passing fluid particle remains constant with time. The path undertaken by a fluid particle under a steady flow is streamline. For example, let us take a non-spinning ball moving relative to a particular fluid. The streamlines around this ball are symmetrical above and below, and the fluid velocity above and below at corresponding points is the same. Thus, there is no pressure difference. The ball moves neither up nor down. Now if we take a similar ball in a fluid which is moving and spinning clockwise. It drags the fluid with it. The ball is moving in the forward direction and relative to it the fluid (air, for example) is moving backward. The fluid velocity above the ball is greater than below. The streamlines get crowded above, resulting in a pressure difference above and below the ball. The result is a net upward force F1. The airfoil shape is such that it can generate this lift when it moves horizontally through a fluid, it is not necessary to spin.

Now, let us look at the various parts of a typical modern airliner. [as show in Figure 2]

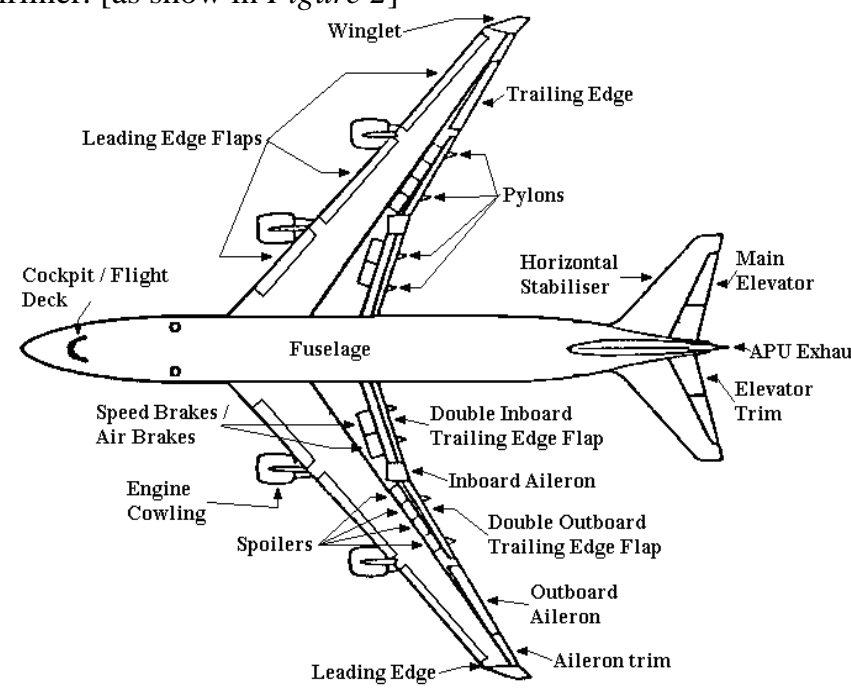

Figure 2: Various parts of a typical modern airliner

C. What about the performance of in-flight maneuvers?

What makes a plane bank, or alter its altitude during flight? Such tasks are performed by certain movable control surfaces and structures called Ailerons, which are present near the end of the main wing, at each side. Alternate movements of ailerons cause the airplane to tilt .The rudder is activated to turn sideways. The rudder is present at the very end of the tail. Sideways movement of the rudder diverts the airflow either left or right. The reaction force pushes the behind portion of the plane in the opposite direction, resulting in the plane turning in the desired direction.

\section{TAKE-OFF}

Take-off, or lift-off, is the most crucial phase of flight. The elevators, located at the trailing edge of the horizontal stabilizers, are moved upward to divert airflow above. The reaction force exerted by the air pushes down the back portion of the airplane. This causes the airplane's nose to tilt upward during the ground run. The velocity at which this happens is known as VR or V-Rotate, since the body of the airplane rotates during this event. Afterward, the plane lifts off. This is the V2 speed. The upward and downward motion of the elevator at the trailing edge is as also responsible for changing altitude

The wings are shaped and designed aerodynamically to generate lift force while kept in fluids (air). In older planes, the wings were designed to be perpendicular to the body. In most modern planes however, the wings are slanted or swept a little backwards. This design actually decreases the lift by a small amount, but increases the speed by a great degree, thus recouping for any small decrease in lift. However, the lift generated by the main wing by itself is insufficient to provide the minimum energy needed to let the aircraft break free from the ground. For this very reason, the wings are equipped with certain movable control surfaces - the leading edge slats and the trailing edge flaps. Before taking off, they are extended downward and as a result the airfoil shape becomes more prominent. The low-hanging flaps also help obstruct and slow down airflow below the main wing. Slower air is denser and is at higher pressure, generating higher lift.

Operating the flaps is intricate, and the flaps are extended to different extents depending on the runway length, weather, mass, etc. [1] Flaps help in maximizing the lift force at a certain angle of attack, without impeding the airplane and avoiding airflow separation. "Angle of attack is the angle between the oncoming air or relative wind and a reference line on the airplane or wing. The higher the angle of attack, the higher is the lift-force. However, there is a limit to this. If the angle of attack is too high, the airflows above and below the wing separate and create turbulent wind vortices. This kills lift and the airplane is unable to climb further. This condition is known as a" stall." 


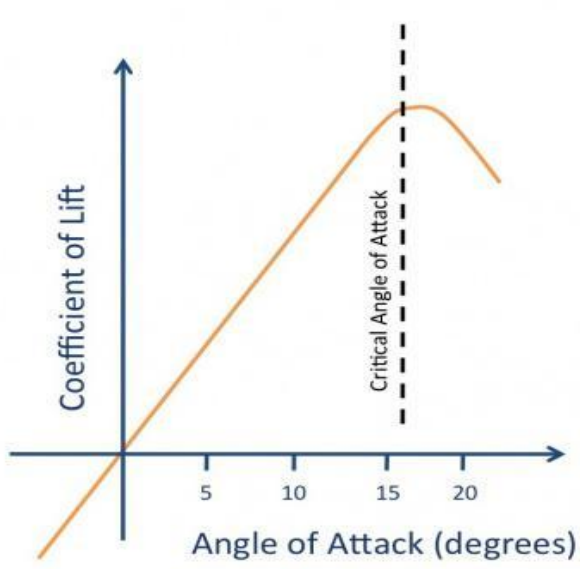

Figure 3: Graph of angle of attack vs. coefficient of lift

Hence, it is clear that the main wing (and the entire airplane, for that matter) is very complex. Even the slightest

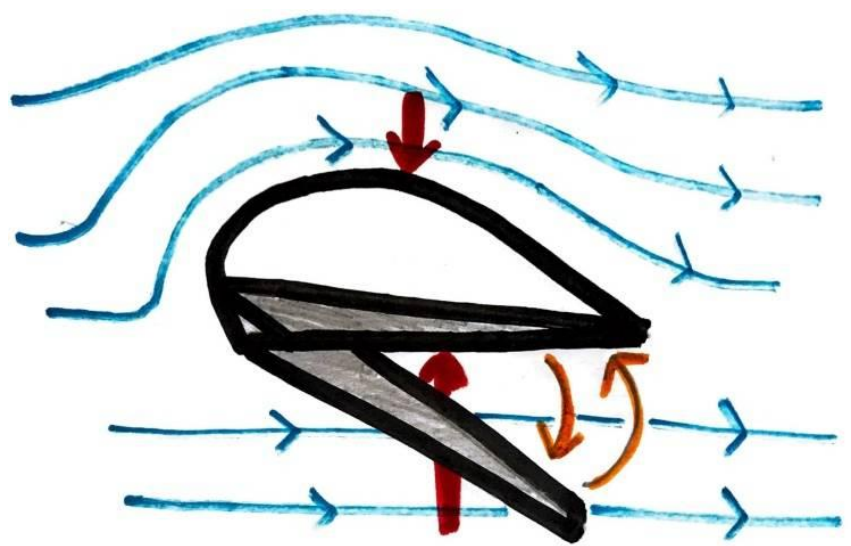

tweak in design can probably cost millions of dollars- but these are only effective as long as they can increase the fuel efficiency and safety of the airplane.

\section{THE "S-FLAP" DESIGN}

From the beginnings of flight, we have devised ingenious ways of improving lift-off efficiency of airplanes. Flaps are used for this very reason. Maximizing the surface area of the wing helps too. Present-Day planes have raked wings and winglets. The wings are curved upward to increase effectiveness. The design proposed is referred to as the "S-Flap" design. It is an auxiliary flap structure in the main wing that would assist rotating at VR speeds during the lift-off. Conventionally, this task is done by the elevator. The "S-Flap" design is essentially a design modification that could be introduced in airplanes to maximize take-off performance and/or pitch performance.

Figure 4: S-Flap Design

In this model, a downward-un-stationary control surface structure is added at the leading edge under the airplane's main wing- near the fuselage. The S-Flap must be added in such a way that it can be folded right within the wing and does not affect the smooth aileron surface structure of the wing. The S-Flap essentially mimics a split flap [2], but is set further forward. Split flaps are not quite efficient and increase drag force to an extent. However, unlike split flaps, the
S-Flap is not meant to be activated for a long time. Thus, the resulting drag would be nearly negligible. The design is more efficient if the aerodyne has a wider wing surface. For instance, in this case, the Antonov would have an advantage over airplanes like the Boeing 737, which has comparatively thinner wings. The S-Flap need not be thick, but should span a large surface area. While the lift-off roll, once the VR Speed is achieved, the S-Flap can be moved down in order to maximize the lift force below the leading edge of the wing. A well-engineered sudden, sharp movement of the S-Flap could provide necessary lift force to the aircraft enabling it to break ground (take flight) without activating the elevator.

While the inconvenience and cost of installing such a surface structure in a commercial plane seems arduous, it can be greatly useful in case of malfunctioning elevators, as well as in assisting in-flight maneuvers during overcast conditions.

Nonetheless, the most significant use of the S-Flap design is that if activated along with the elevators, it could decrease lift-off space and/or maximize lift-off weight of any craft appreciably.

The vital concern is that this design considerably affects the fuel capacity as the fuel in most aerodynes is stored inside the main wing. But the fuel could be stored inside the S-Flap too, and joining the point between the main wings and the $\mathrm{S}$-Flap, the pivot, could serve as the fuel pipe from the S-Flap to the wings, in turn to the engines

In case of short runways or other emergency conditions, the aircraft already at lift-off roll, could easily take-off with the help of the S-Flap even below VR speed rather than aborting the entire procedure, which at times becomes nearly impossible. With such a structure, accidents like runway overruns during lift-offs can be avoided. It may also prove to be efficient in restraining turbulence, and with a balanced connection to the lift spoilers on the wings, the horizontal stabilizers may not need any movable surface. But, the horizontal stabilizers must be there for in-flight stability.

The S-Flap design would be very convenient to install in crafts with high wings/flaps. Most commercial planes are low-winged. Some airplanes, like regional jets and cargo airplanes, have the main wing mounted over the body; they are high-winged. [3]The "center of mass" (COM) is beneath the "center of lift" in a high-wing airplane, resulting in much more stability as compared to a low-winged airplane (centre of mass is above the centre of lift). To improve the stability of a low-wing aircraft, designers recoup by curving the wingtips upward (dihedral).

Apart from the costs involved, there is no direct problem with the S-Flap design. The question of airflow separation is also irrelevant if the S-Flap is lowered for a small instant. Using both the proposed S-Flap and the elevator together increases the risk of tail strike. Additionally, the most important question is the particular angle by which the S-Flap needs to be lowered. In planes that are low-winged this might be a problem - since the risk of the S-Flap touching/scratching the runway is present. Fortunately, present day airplanes usually have good ground clearance. 
Also, an important point to note is that low-wing airplanes have minimized elevator and rudder efficiency, as the main wing blocks some air-flow to the rear end of the plane. This substantiates the installation of the S-Flap structure to assist elevators and maximize the efficiency of maneuvers.

The aircraft spoilers could also be shaped as an Upside-Down (U-D) aileron. This would maximize the rearward drag force if the wind is from an upward direction, that is, downward and to some extent, if the wind is from downward, too. This minute modification in the design of the lift spoilers should neither cost much, nor be inconvenient. The practical effectiveness of such a design needs to be experimentally tested, nevertheless. To conclude, it is evident that the slightest design modification in the main wing of the craft can work miraculously in increasing the efficiency of aerodynes

\section{CONCLUSION}

It can thus be deduced that adding a downward-movable surface structure at the leading head of the main wing in an aerodyne may help in increasing the lift-off efficiency in-flight pitch control. It may also prove to be very helpful in certain crises situations. Despite its high cost, such a structure could help planes lift-off well below typical speed in short runways and adverse conditions. Aspects such as ground clearance, wing arrangement, engine positioning etc., need to be taken into account before installing such a structure. Nonetheless, such a design modification is possible in almost any airplane. It should be preferable to install such a structure in high-winged airplanes with good ground clearance, and with engines that require the horizontal stabilizer be above the engines.

\section{REFERENCES}

[1] Aviation Stack Exchange. https://aviation.stackexchange.com/

[2] Dr. Scholz. "High Lift Systems and Maximum Lift Coefficients". Aircraft Design - an Open Educational Resource (OER) for Hamburg Open Online University (HOOU) https://www.fzt.haw-hamburg.de/pers/Scholz/HOOU/AircraftDesign_ 8_HighLift.pdf

[3] "High Wing vs Low Wing: What's the Difference between Them?" Pilot Mall. 2020 https://www.pilotmall.com/blogs/news/high-wing-vs-low-wing-what-s -the-difference-between-them

[4] https://www.princeton.edu/ asmits/Bicycle_web/continuity.html 